\title{
THE CONLEY INDEX AND COUNTABLE DECOMPOSITIONS OF INVARIANT SETS
}

\author{
MARIAN GIDEA \\ Department of Mathematical and Computer Sciences \\ Loyola University of Chicago \\ 6525 North Sheridan Road \\ Chicago, Illinois 60626, U.S.A. \\ E-mail: mgidea@math.luc.edu
}

Dedicated to the memory of my father

\begin{abstract}
We define a new cohomological index of Conley type associated to any bi-infinite sequence of neighborhoods that satisfies a certain isolation condition. We use this index to study the chaotic dynamics on invariant sets which decompose as countable unions of pairwise disjoint $(\bmod 0)$ compact pieces.
\end{abstract}

1. Introduction. The construction of the Conley index for discrete time dynamical systems ([Mr1], [Mr2]) has been followed by an impressive number of results and applications in detecting chaotic behavior. Some of the advantages of using the Conley index are the easy computability and the relatively strong description of the dynamics (in terms of semiconjugacy to a shift space) it provides.

The desire of obtaining more and more accurate information on a wider range of discrete dynamical systems displaying complicated behavior has led to the construction of numerous versions of the index [DeMr], [MiMr1], [Sz1], [Sz2], [Gi1], [Gi2], [Gi3]. Dealing with a large number of fairly abstract Conley index types of invariants rather than a simple one, as the original index intended to be, may seem frustrating. On the other hand, the new techniques have paid off, in the sense of providing very precise descriptions of chaotic systems.

In [Sz], Szymczak introduces a new technique of detection of chaos based on the construction of the Conley index for decompositions of isolated invariant sets. He defines an index for isolated invariant sets which admit decompositions as disjoint unions of finitely many compact sets. His index can detect chaos by showing the existence of a

1991 Mathematics Subject Classification: Primary 58F35; Secondary 34C35.

The paper is in final form and no version of it will be published elsewhere. 
semiconjugacy between the map itself (rather than a certain iterate of the map, like other previous results [MiMr2], [CaKwMi1], [CaKwMi2]) and the one-sided shift map on the shift space that has as many symbols as the components of the decomposition.

The purpose of this paper is to adapt some of the techniques developed in [Gi1], [Gi2], [Gi3], in order to study countable decompositions of invariant sets which are not necessarily isolated. The main example is the case of hyperbolic systems with singularities (billiard systems) which we may study only from a topological point of view. We generally assume the existence of a decomposition of a certain neighborhood of an invariant set into countably many pairwise disjoint $(\bmod 0)$ and compact pieces. Then we can compute a cohomological invariant of Conley type for each bi-infinite sequence of arbitrary "neighborhoods" from the decomposition. The fundamental properties usually satisfied by the Conley index hold for this invariant, too.

It may seem unreasonable to compute the index of infinitely many bi-infinite sequences of neighborhoods in order to detect chaotic behavior! However, considerably less information is sufficient to obtain a local semiconjugacy to a shift space. Due to the fact that the invariant sets we consider may fail to be isolated, our approach is essentially different from that of Szymczak [Sz1]. In the case of an isolated invariant set, both techniques provide the same information about the chaotic behavior of the system.

2. The construction of the index. Assume that $X$ is a locally compact metric space, $f: X \rightarrow X$ a homeomorphism and $S$ a subset of $X$ invariant with respect to $f$. Note that $S$ is assumed to be neither isolated nor compact. We say that two subsets $A$ and $B$ of $X$ are disjoint $(\bmod 0)$ if the intersection $A \cap B$ is nowhere dense. We will denote by $H^{*}$ the Alexander-Spanier cohomology functor with coefficients in $R$, a principal ideal domain and by $\mathcal{L}$ the "extended" Leray functor from the category of graded directed systems of modules and homomorphisms into its proper subcategory of graded directed systems of modules and isomorphisms (see [Mr1] and [Gi2]) for details).

If $\left\{V_{i}\right\}_{i \in \mathbf{Z}}$ is a sequence of subsets of $X$, for each $i \in \mathbf{Z}$ and $n \in \mathbf{N}$ let

$$
O I\left(V_{i}\right)=\bigcap_{k \in \mathbf{Z}} f^{-k}\left(V_{i+k}\right)
$$

and

$$
O I^{n}\left(V_{i}\right)=\bigcap_{-n \leq k \leq n} f^{-k}\left(V_{i+k}\right)
$$

Let $\mathcal{V}$ denote a countable family of pairwise disjoint $(\bmod 0)$ compact subsets of $X$ that covers $S$ and satisfies the following property:

$$
\begin{aligned}
& \text { there exists } n \in \mathbf{N} \text { such that for any bi-infinite sequence }\left\{V_{i}\right\}_{i \in \mathbf{Z}} \text { of elements of } \mathcal{V} \\
& \qquad O I^{n}\left(V_{i}\right) \subseteq i n t V_{i} \text {, for all } i \in \mathbf{Z} .
\end{aligned}
$$

Any such a $\left\{V_{i}\right\}_{i}$ is called an isolating neighborhood sequence. The collection of all isolating neighborhood sequences will be denoted by $\mathcal{W}$.

The following lemma is obvious, so we omit the proof.

LEMMA 2.1. If $\left\{V_{i}\right\}_{i \in \mathbf{Z}}$ and $\left\{W_{i}\right\}_{i \in \mathbf{Z}}$ are isolating neighborhood sequences then (1) If $V_{i} \subseteq W_{i}$ for all $i$ then $O I^{n}\left(V_{i}\right) \subseteq O I^{n}\left(W_{i}\right)$ for all $i$ and $n$. 
(2) If $n_{1} \geq n_{2} \geq 0$ then $O I^{n_{1}}\left(V_{i}\right) \subseteq O I^{n_{2}}\left(V_{i}\right)$ for all $i$.

(3) If $n_{1}, n_{2} \geq 0, O I^{n_{1}+n_{2}}\left(V_{i}\right)=O I^{n_{1}}\left(O I^{n_{2}}\left(V_{i}\right)\right)$ for all $i$.

(4) If $n_{1}, n_{2} \geq 0, O I^{n_{1}}\left(V_{i}\right) \subseteq i n t V_{i}$ for all $i$, then $O I^{n_{1}+n_{2}}\left(V_{i}\right) \subseteq \operatorname{int}\left(O I^{n_{2}}\left(V_{i}\right)\right)$ for all $i$.

(5) $O I^{n}\left(V_{i}\right)$ is compact for all $i$ and $n$.

Definition 2.2. A sequence of compact pairs $\left(N_{i}, L_{i}\right)_{i \in \mathbf{Z}}$ with $L_{i} \subset N_{i} \subset X$ is called an index pair sequence if the following properties are satisfied for each $i \in \mathbf{Z}$ :

(1) There exists $n \geq 0$ such that $O I^{n}\left(\operatorname{cl}\left(N_{i} \backslash L_{i}\right)\right) \subseteq \operatorname{int}\left(N_{i} \backslash L_{i}\right)$ for some $n \in \mathbf{N}$ and all $i \in \mathbf{Z}$, thus $\left\{c l\left(N_{i} \backslash L_{i}\right)\right\}_{i}$ is an isolating neighborhood sequence.

(2) $f\left(L_{i}\right) \cap N_{i+1} \subseteq L_{i+1}$.

(3) $f\left(N_{i} \backslash L_{i}\right) \subseteq N_{i+1}$.

We say that $\left(N_{i}, L_{i}\right)_{i}$ is an index pair sequence for $\left\{V_{i}\right\}_{i}$ if the following condition holds instead of $(1)$ ( in particular $\left(1^{\prime}\right)$ implies (1)):

$\left(1^{\prime}\right) O I^{n}\left(V_{i}\right) \subset \operatorname{int}\left(N_{i} \backslash L_{i}\right) \subset N_{i} \subset \operatorname{int} V_{i}$ for some $n \in \mathbf{N}$ and all $i \in \mathbf{Z}$.

REMARK 2.3. If $S$ is an isolated invariant set, $V$ is an isolating neighborhood and $(N, L)$ is an index pair for $S$, then we may set $V_{i}:=V$ and $\left(N_{i}, L_{i}\right):=(N, L)$ for all $i \in \mathbf{Z}$, obtaining $\left\{V_{i}\right\}_{i}$ an isolating neighborhood sequence and $\left(N_{i}, L_{i}\right)_{i}$ an index pair sequence for $\left\{V_{i}\right\}_{i}$ (the condition $O I^{n}\left(\operatorname{cl}\left(N_{i} \backslash L_{i}\right)\right) \subset \operatorname{int}\left(N_{i} \backslash L_{i}\right)$ for some $n$ is easily satisfied since $X$ is a locally compact metric space). Therefore, we can say that isolating neighborhoods and index pairs are particular cases of isolating neighborhood sequences and index pair sequences, respectively.

THEOREM 2.4 (Existence of index pair sequences). If $\left\{V_{i}\right\}_{i}$ is an isolating neighborhood sequence then there exists $\left(N_{i}, L_{i}\right)_{i}$ an index pair sequence for $\left\{V_{i}\right\}_{i}$.

Proof. Since $\left\{V_{i}\right\}_{i}$ is an isolating neighborhood sequence, there exists $n \in \mathbf{N}$ such that $O I^{n}\left(V_{i}\right) \subseteq \operatorname{int} V_{i}$, for all $i \in \mathbf{Z}$. Define:

$$
N_{i}:=O I^{n}\left(V_{i}\right)
$$

$L_{i}=O L^{n}\left(V_{i}\right):=\left\{x \in N_{i} \mid\right.$ there exists $k, 0 \leq k \leq n+1$, such that $\left.f^{k}(x) \notin i n t V_{i+k}\right\}$

for each $i \in \mathbf{Z}$. We would like to check that $\left(N_{i}, L_{i}\right)_{i}$ satisfies the axioms $\left(1^{\prime}\right),(2)$ and (3) from Definition 2.2, so it constitutes an index pair sequence.

$$
N_{i} \backslash L_{i}=\bigcap_{-n \leq k \leq-1} f^{-k}\left(V_{i+k}\right) \cap \bigcap_{0 \leq k \leq n+1} f^{-k}\left(i n t V_{i+k}\right) .
$$

By Lemma 2.1, $O I^{2 n+1}\left(V_{i}\right) \subseteq O I^{n+1}\left(\operatorname{int} V_{i}\right) \subseteq \operatorname{int}\left(N_{i} \backslash L_{i}\right)$. On the other hand, it is clear that $N_{i} \subseteq \operatorname{int} V_{i}$, thus $\left(1^{\prime}\right)$ is verified.

(2) If $y \in f\left(L_{i}\right) \cap N_{i+1}$, then $y=f(x)$ for some $x \in L_{i}$. Since $y \in N_{i+1}$, either $y \in N_{i+1} \backslash L_{i+1}$ or $y \in L_{i+1}$. If $y \in N_{i+1} \backslash L_{i+1}$ then

$$
x \in \bigcap_{-n+1 \leq k \leq 0} f^{-k}\left(V_{i+k}\right) \cap \bigcap_{1 \leq k \leq n+2} f^{-k}\left(i n t V_{i+k}\right) .
$$

On the other hand, since $x \in L_{i}, f^{k}(x) \notin V_{i+k}$ for some $k \in\{0,1, \ldots n-1\}$. It follows that $x=f^{0}(x) \notin \operatorname{int} V_{i}$, which is a contradiction to $x \in L_{i} \subseteq N_{i} \subseteq$ int $V_{i}$. 
(3) If $y=f\left(N_{i} \backslash L_{i}\right)$ then $y=f(x)$ for some $x \in N_{i} \backslash L_{i}$, so

$$
f(x) \in \bigcap_{-n-1 \leq k \leq-2} f^{-k}\left(V_{i+1+k}\right) \cap \bigcap_{-1 \leq k \leq n} f^{-k}\left(i n t V_{i+1+k}\right)
$$

thus $y=f(x) \in O I^{n}\left(V_{i+1}\right)=N_{i+1}$.

ExAMPLE 2.5. The Smale's U-horseshoe is obtained by continuously transforming the square $Q$ of vertices $A, B, C, D$ into the horseshoe shaped region of vertices $f(A), f(B)$, $f(C), f(D)$ as in Figure 2.1. The two components of $f^{-1}(Q) \cap Q$ are denoted by $V_{0}$ and $V_{1}$. There are no extra assumptions of uniformly stretching and shrinking of the edges but we do require that:

$$
f^{-1}\left(V_{i}\right) \cap V_{j} \cap f\left(V_{k}\right) \subseteq i n t V_{j}
$$

for any choice of $i, j, k \in\{0,1\}$ (these conditions are obviously satisfied if we impose hyperbolicity on the dynamical system). Under this condition, the invariant set $S$ of $Q$ is an isolated invariant set (not necessarily a Cantor set) and its cover is $\mathcal{V}=\left\{V_{0}, V_{1}\right\}$.

If $a=\left(a_{i}\right)_{i \in \mathbf{Z}}$ is any bi-infinite sequence of symbols in $\{0,1\}$, then $\left\{V_{a_{i}}\right\}_{i \in \mathbf{Z}}$ is an isolating neighborhood sequence: for $n=1, O I^{n}\left(V_{a_{i}}\right) \subseteq i n t V_{a_{i}}$ due to (2.2). Now define $N_{a_{i}}=V_{a_{i}}$ and $L_{a_{i}}=N_{a_{i}} \backslash f^{-1}\left(i n t N_{a_{i+1}}\right)$ for all $i \in \mathbf{Z}$. This makes $\left(N_{a_{i}}, L_{a_{i}}\right)_{i \in \mathbf{Z}}$ an index pair sequence for $\left\{V_{a_{i}}\right\}_{i \in \mathbf{Z}}$.

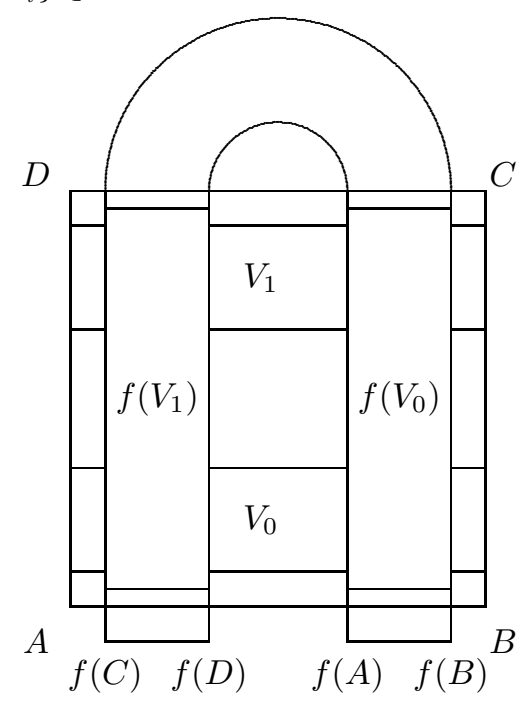

Fig. 2.1. The U-horseshoe

EXAMPLE 2.6. We will consider a dispersed billiard in a two-dimensional domain of $\mathbf{R}^{2}$ or of the two-dimensional torus (see [BuSi], [Bu]). Let $Q$ denote a two-dimensional open bounded connected domain on $\mathbf{R}^{2}$ or on the two-dimensional torus with Euclidean metric, whose boundary is a finite union of smooth non-self-intersecting curves either closed or having common end-points. There exists a framing of each boundary curve by unit normal vectors pointing inside $Q$. For a dispersed billiard, by definition, the curvature of each component of the boundary is strictly positive. We will consider the dynamical 


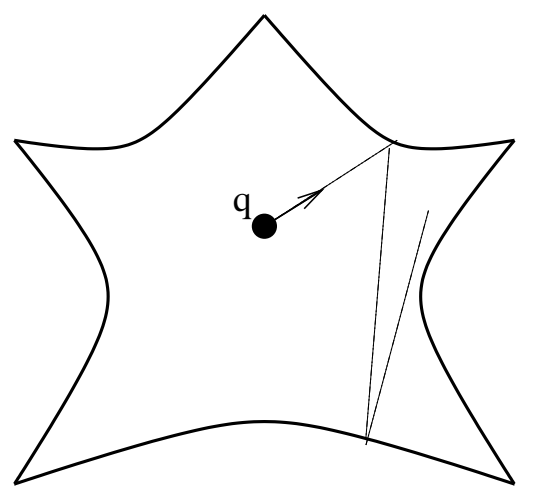

Fig. 2.2. The dispersed billiard system

system corresponding to the inertial motion of a particle inside $Q$ with elastic reflection at the boundary (see Figure 2.2.).

This is modeled as follows. Let $M$ be the unit tangent bundle over $Q, \pi: M \rightarrow Q$ the natural projection and $M_{1}$ the set of all points $x$ of the boundary of $M$ such that $x$ is not orthogonal to $n(q)$, where $q=\pi(x)$. The position $q$ and the velocity $v$ of the particle at the boundary are described by a unit vector $x=(q, v) \in M_{1}$, while $T(x)$ represents position and velocity in the instant right after the first reflection. We may restrict ourselves to $M_{1}$ in order to obtain $T: M_{1} \rightarrow M_{1}$ a well defined map. Bunimovich and Sinai have proved the existence of a countable Markov partition for dispersed billiards (see [BuSi]). The invariant set $S$ of $M$ (or of $M_{1}$ ) is not isolated. Let us denote $\mathcal{V}=\left\{V_{1}, V_{2}, \ldots, V_{n}, \ldots\right\}$ the collection of all of the rectangles of the partition. If $a=\left(a_{i}\right)_{i \in \mathbf{Z}}$ is a bi-infinite sequence of symbols in $\{1,2, \ldots, n, \ldots\}$ then $\left\{V_{a_{i}}\right\}_{i \in \mathbf{Z}}$ is an isolating neighborhood sequence: for $m=1, O I^{m}\left(V_{a_{i}}\right) \subseteq \operatorname{int} V_{a_{i}}$ for all $i \in \mathbf{Z}$. Again we can define $N_{a_{i}}=V_{a_{i}}$ and $L_{a_{i}}=$ $N_{a_{i}} \backslash f^{-1}\left(i n t N_{a_{i+1}}\right)$ obtaining $\left(N_{a_{i}}, L_{a_{i}}\right)_{i}$ an index pair sequence for $\left\{V_{a_{i}}\right\}_{i \in \mathbf{Z}}$.

The same conclusion holds if we assume that $\mathcal{V}$ represents only a partition of $M_{1}$ into countably many pairwise disjoint $(\bmod 0)$ compact pieces satisfying the condition:

$$
f^{-1}\left(V_{i}\right) \cap V_{j} \cap f\left(V_{k}\right) \subseteq \operatorname{int} V_{j}
$$

for any choice of $i, j, k \in\{1,2, \ldots, n, \ldots\}$, without being properly a Markov partition.

In the sequel, we construct a Conley index type of invariant for each isolating neighborhood sequence generated by the decomposition $\mathcal{V}$, in order to obtain a detailed description of the dynamics on $S$.

On $\mathcal{W}$ we define an equivalence relation: $\left\{V_{i}\right\}_{i \in \mathbf{Z}} \sim\left\{V_{i}^{\prime}\right\}_{i \in \mathbf{Z}}$ if there exists $m>0$ such that $O I^{m}\left(V_{i}\right) \subseteq i n t V_{i}^{\prime}$ and $O I^{m}\left(V_{i}^{\prime}\right) \subseteq i n t V_{i}$ for all $i \in \mathbf{Z}$. We will denote the equivalence class of $\left\{V_{i}\right\}_{i}$ in $\mathcal{W}$ by $\left[\left\{V_{i}\right\}_{i}\right]$. Two index pairs $\left(N_{i}, L_{i}\right)_{i}$ and $\left(N_{i}^{\prime}, L_{i}^{\prime}\right)_{i}$ are said to be equivalent, $\left(N_{i}, L_{i}\right)_{i} \sim\left(N_{i}^{\prime}, L_{i}^{\prime}\right)_{i}$, if there exists $m>0$ such that $O I^{m}\left(\operatorname{cl}\left(N_{i} \backslash L_{i}\right)\right) \subseteq$ $\operatorname{int}\left(N_{i}^{\prime} \backslash L_{i}^{\prime}\right)$ and $O I^{m}\left(\operatorname{cl}\left(N_{i}^{\prime} \backslash L_{i}^{\prime}\right)\right) \subseteq \operatorname{int}\left(N_{i} \backslash L_{i}\right)$ for all $i \in \mathbf{Z}$. It follows that, if $\left(N_{i}, L_{i}\right)_{i}$ and $\left(N_{i}^{\prime}, L_{i}^{\prime}\right)_{i}$ are two index pair for the same $\left\{V_{i}\right\}_{i} \in \mathcal{W}$, then $\left(N_{i}, L_{i}\right)_{i} \sim\left(N_{i}^{\prime}, L_{i}^{\prime}\right)_{i}$. Moreover, if $\left(N_{i}, L_{i}\right)_{i}$ is an index pair for $\left\{V_{i}\right\}_{i},\left(N_{i}^{\prime}, L_{i}^{\prime}\right)_{i}$ is an index pair for $\left\{V_{i}^{\prime}\right\}_{i}$ and $\left\{V_{i}\right\}_{i} \sim\left\{V_{i}^{\prime}\right\}_{i}$, then $\left(N_{i}, L_{i}\right)_{i} \sim\left(N_{i}^{\prime}, L_{i}^{\prime}\right)_{i}$. 
Now consider an equivalence class $\left[\left\{V_{i}\right\}_{i}\right] \in W / \sim$, an isolating neighborhood sequence $\left\{V_{i}\right\}_{i} \in\left[\left\{V_{i}\right\}_{i}\right]$ and an index pair sequence $\left(N_{i}, L_{i}\right)_{i}$ for $\left\{V_{i}\right\}_{i}$. We proceed to the following construction:

Step 1. Consider the restriction of $f$ :

$$
f_{N_{i}}:\left(N_{i}, L_{i}\right) \longrightarrow\left(N_{i+1} \cup f\left(N_{i}\right), L_{i+1} \cup f\left(L_{i}\right)\right)
$$

which induces the homomorphism

$$
f_{N_{i}}^{*}: H^{*}\left(\left(N_{i+1} \cup f\left(N_{i}\right), L_{i+1} \cup f\left(L_{i}\right)\right) \rightarrow H^{*}\left(N_{i}, L_{i}\right) .\right.
$$

Step 2. By the condition (2) of Definition 2.2 and the strong excision property for the Alexander-Spanier cohomology (see [Sp], p. 318), the inclusion

$$
j_{N_{i}}:\left(N_{i}, L_{i}\right) \rightarrow\left(N_{i+1} \cup f\left(N_{i}\right), L_{i+1} \cup f\left(L_{i}\right)\right.
$$

induces an isomorphism

$$
j_{N_{i}}^{*}: H^{*}\left(\left(N_{i+1} \cup f\left(N_{i}\right), L_{i+1} \cup f\left(L_{i}\right)\right) \rightarrow H^{*}\left(N_{i}, L_{i}\right) .\right.
$$

Step 3. Now we define the transfer map

$$
F_{N_{i}}^{*}: H^{*}\left(N_{i+1}, L_{i+1}\right) \rightarrow H^{*}\left(N_{i}, L_{i}\right),
$$

where $F_{N_{i}}^{*}=f_{N_{i}}^{*} \circ\left(j_{N_{i}}^{*}\right)^{-1}$.

At this point we have obtained a system of $R$-modules and connecting homomorphisms

$$
\ldots \longrightarrow H^{*}\left(N_{i+1}, L_{i+1}\right) \stackrel{F_{N_{i}}^{*}}{\longrightarrow} H^{*}\left(N_{i}, L_{i}\right) \stackrel{F_{N_{i-1}}^{*}}{\longrightarrow} H^{*}\left(N_{i-1}, L_{i-1}\right) \longrightarrow \ldots
$$

Now we apply a Leray functor and we obtain

$$
\ldots \longrightarrow M_{i+1} \stackrel{\chi_{i}^{*}}{\longrightarrow} M_{i} \stackrel{\chi_{i-1}^{*}}{\longrightarrow} M_{i-1} \longrightarrow \ldots
$$

where all $M_{i}$ are $R$-modules and $\chi_{i}^{*}$ are $R$-module isomorphisms.

Consider the direct $\operatorname{limit} \lim M_{i}$ of the modules $M_{i}$ which is isomorphic to each of the $M_{i}$ and let $\lim _{\rightarrow} \chi_{i}^{*}$ be any one of the automorphisms of $\lim _{\rightarrow} M_{i}$ induced by the isomorphisms $\chi_{i}^{*}$.

Definition 2.7. The Conley index $\operatorname{Con}^{*}\left(\left[\left\{V_{i}\right\}_{i}\right]\right)$ for $\left[\left\{V_{i}\right\}_{i}\right]$ is defined to be either the directed system of $R$-modules and isomorphisms in (2.5)

$$
\ldots \longrightarrow M_{i+1} \stackrel{\chi_{i}^{*}}{\longrightarrow} M_{i} \stackrel{\chi_{i-1}^{*}}{\longrightarrow} M_{i-1} \longrightarrow \ldots
$$

or the pair

$$
\left(C H^{*}\left(\left[\left\{V_{i}\right\}_{i}\right]\right), \chi^{*}\left[\left\{V_{i}\right\}_{i}\right]\right):=\left(\lim _{\rightarrow} M_{i}, \lim _{\rightarrow} \chi_{i}^{*}\right)
$$

and extends naturally to a definition of the index for the isolating neighborhood sequence $\left\{V_{i}\right\}_{i}$ and for the index pair sequence $\left(N_{i}, L_{i}\right)_{i}$.

TheOrem 2.8 (The Conley index is well defined). If $\left(N_{i}, L_{i}\right)_{i}$ is an index pair sequence for $\left\{V_{i}\right\}_{i} \in\left[\left\{V_{i}\right\}_{i}\right]$ and $\left(N_{i}^{\prime}, L_{i}^{\prime}\right)$ is an index pair sequence for $\left\{V_{i}^{\prime}\right\}_{i} \in\left[\left\{V_{i}\right\}_{i}\right]\left(\left\{V_{i}\right\}_{i} \sim\right.$ $\left.\left\{V_{i}^{\prime}\right\}_{i}\right)$ then $\operatorname{Con}^{*}\left(\left(N_{i}, L_{i}\right)_{i}\right)$ and $\operatorname{Con}^{*}\left(\left(N_{i}^{\prime}, L_{i}^{\prime}\right)_{i}\right)$ are isomorphic.

Proof. Step 1. Assume that $\left(N_{i}, L_{i}\right)_{i}$ and $\left(N_{i}^{\prime}, L_{i}^{\prime}\right)_{i}$ satisfy the following conditions:

$$
\left(N_{i}, L_{i}\right) \subseteq\left(N_{i}^{\prime}, L_{i}^{\prime}\right) \quad \text { for all } i \in \mathbf{Z}
$$




$$
f\left(N_{i}^{\prime} \backslash L_{i}^{\prime}\right) \subseteq N_{i+1} \cup f\left(N_{i}\right) \quad \text { for all } i \in \mathbf{Z}
$$

Note that the condition

$$
N_{i}^{\prime} \backslash L_{i}^{\prime} \subseteq N_{i} \backslash L_{i} \quad \text { for all } i \in \mathbf{Z}
$$

implies the condition (2.8).

The inclusion map

$$
k_{i}:\left(\left(N_{i}^{\prime} \backslash L_{i}^{\prime}\right) \cup L_{i}, L_{i}\right) \longrightarrow\left(N_{i}^{\prime}, L_{i}^{\prime}\right)
$$

induces an isomorphism due to the strong excision property of the Alexander-Spanier cohomology

$$
k_{i}^{*}: H^{*}\left(N_{i}^{\prime}, L_{i}^{\prime}\right) \longrightarrow H^{*}\left(\left(N_{i}^{\prime} \backslash L_{i}^{\prime}\right) \cup L_{i}, L_{i}\right) .
$$

Now define the mapping

$$
f_{N_{i}^{\prime}, N_{i+1}}:\left(\left(N_{i}^{\prime} \backslash L_{i}^{\prime}\right) \cup L_{i}, L_{i}\right) \longrightarrow\left(N_{i+1} \cup f\left(N_{i}\right), L_{i+1} \cup f\left(L_{i}\right)\right)
$$

which is well defined by (2.8) and induces the homomorphism

$$
f_{N_{i}^{\prime}, N_{i+1}}^{*}: H^{*}\left(N_{i+1} \cup f\left(N_{i}\right), L_{i+1} \cup f\left(L_{i}\right)\right) \longrightarrow H^{*}\left(\left(N_{i}^{\prime} \backslash L_{i}^{\prime}\right) \cup L_{i}, L_{i}\right) .
$$

Also consider

$$
f_{N_{i}^{\prime}} \circ k_{i}:\left(\left(N_{i}^{\prime} \backslash L_{i}^{\prime}\right) \cup L_{i}, L_{i}\right) \longrightarrow\left(N_{i+1}^{\prime} \cup f\left(N_{i}^{\prime}\right), L_{i+1}^{\prime} \cup f\left(L_{i}^{\prime}\right)\right)
$$

which induces the homomorphism

$$
k_{i}^{*} \circ f_{N_{i}^{\prime}}^{*}: H^{*}\left(N_{i+1}^{\prime} \cup f\left(N_{i}^{\prime}\right), L_{i+1}^{\prime} \cup f\left(L_{i}^{\prime}\right)\right) \longrightarrow H^{*}\left(\left(N_{i}^{\prime} \backslash L_{i}^{\prime}\right) \cup L_{i}, L_{i}\right) .
$$

To simplify notation, we set up $\left(\widehat{N}_{i+1}, \widehat{L}_{i+1}\right)_{i}:=\left(N_{i+1} \cup f\left(N_{i}\right), L_{i+1} \cup f\left(L_{i}\right)\right)_{i},\left(\widehat{N}_{i+1}^{\prime}, \widehat{L}_{i+1}^{\prime}\right)_{i}$ $:=\left(N_{i+1}^{\prime} \cup f\left(N_{i}^{\prime}\right), L_{i+1}^{\prime} \cup f\left(L_{i}^{\prime}\right)\right)_{i}$ and $\left(\widetilde{N}_{i}^{\prime}, \widetilde{L}_{i}^{\prime}\right):=\left(\left(N_{i}^{\prime} \backslash L_{i}^{\prime}\right) \cup L_{i}, L_{i}\right)_{i}$.

We have the following commutative diagram with descending vertical arrows inclusion maps:

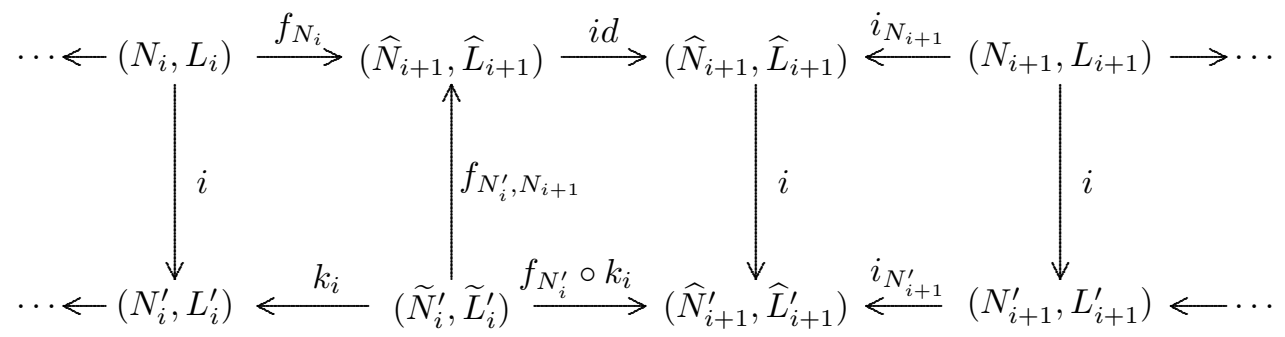

The cohomology functor $H^{*}$ applied to the above diagram produces the following commutative diagram:

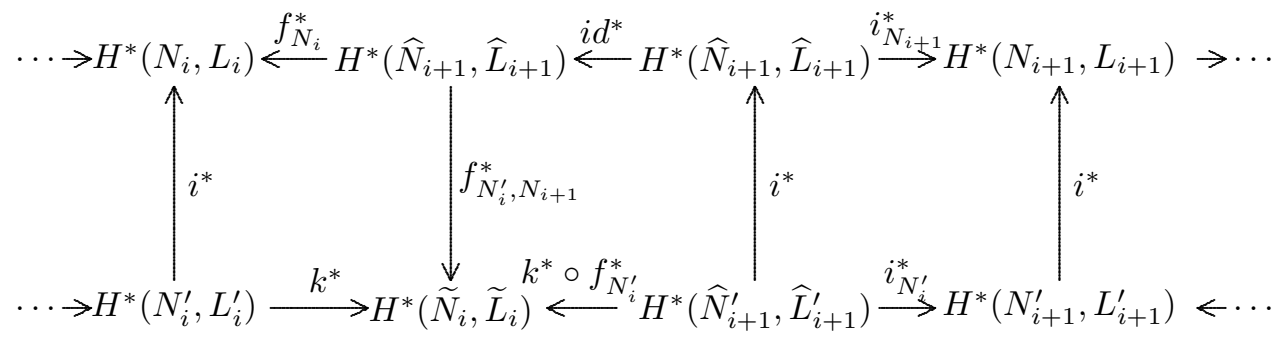


After removing $H^{*}\left(\widehat{N}_{i+1}, \widehat{L}_{i+1}\right), H^{*}\left(\widehat{N}_{i+1}^{\prime}, \widehat{L}_{i+1}^{\prime}\right)$ and $H^{*}\left(\widetilde{N}_{i}, \widetilde{L}_{i}\right)$ from the above diagram, we obtain:

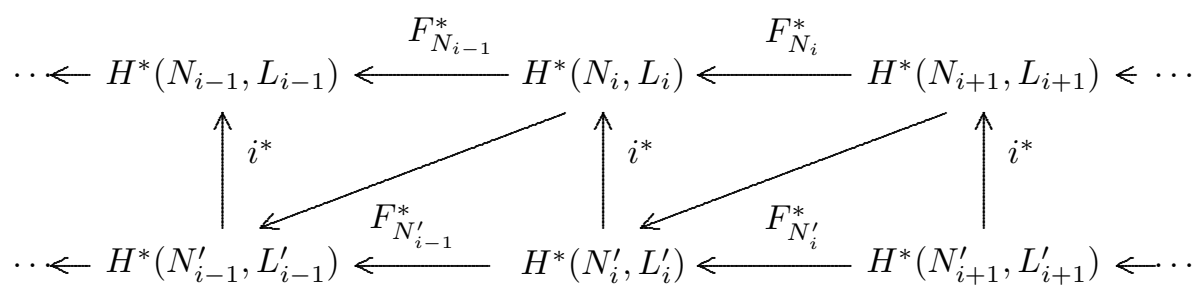

where the slanted arrows are the homomorphisms $\left(k_{i-1}^{*}\right)^{-1} \circ f_{N_{i-1}^{\prime}, N_{i}}^{*} \circ\left(i_{N_{i}}^{*}\right)^{-1}$ and $\left(k_{i}^{*}\right)^{-1} \circ$ $f_{N_{i}^{\prime}, N_{i+1}}^{*} \circ\left(i_{N_{i+1}}^{*}\right)^{-1}$, respectively.

According to $[\mathrm{Gi} 2]$ the objects $\left(H^{*}\left(N_{i}, L_{i}\right), F_{N_{i}}^{*}\right)_{i}$ and $\left(H^{*}\left(N_{i}^{\prime}, L_{i}^{\prime}\right), F_{N_{i}^{\prime}}^{*}\right)_{i}$ are linked, thus $\mathcal{L}\left(\left(H^{*}\left(N_{i}, L_{i}\right), F_{N_{i}}^{*}\right)_{i}\right)$ and $\mathcal{L}\left(\left(H^{*}\left(N_{i}^{\prime}, L_{i}^{\prime}\right), F_{N_{i}^{\prime}}^{*}\right)_{i}\right)$ are isomorphic.

Step 2. Assume that $\left(N_{i}, L_{i}\right)_{i}$ and $\left(N_{i}^{\prime}, L_{i}^{\prime}\right)_{i}$ satisfy the following conditions:

$$
\left(N_{i}, L_{i}\right) \subseteq\left(N_{i}^{\prime}, L_{i}^{\prime}\right) \text { for all } i \in \mathbf{Z}
$$

$$
L_{i}=L_{i}^{\prime} \text { for all } i \in \mathbf{Z}
$$

Define the index pair sequences $\left\{\left(A_{i}^{k}, B_{i}^{k}\right)_{i \in \mathbf{Z}}\right\}_{k \in \mathbf{N}}$ by:

$$
\begin{gathered}
A_{i}^{0}=N_{i}^{\prime} \\
B_{i}^{0}=L_{i} \cup\left(f^{-1}\left(L_{i+1}\right) \cap N_{i}^{\prime}\right) \\
A_{i}^{2 k}=N_{i} \cup O I^{k}\left(N_{i}^{\prime}\right) \\
B_{i}^{2 k}=L_{i} \cup\left(\bigcap_{1 \leq j \leq k} f^{-j}\left(L_{i+j}\right) \cap O I^{k}\left(N_{i}^{\prime}\right)\right) \\
A_{i}^{2 k+1}=N_{i} \cup\left(f^{-(k+1)}\left(N_{i+k+1}^{\prime}\right) \cap O I^{k}\left(N_{i}^{\prime}\right)\right) \\
B_{i}^{2 k+1}=L_{i} \cup\left(\bigcap_{1 \leq j \leq k+1} f^{-j}\left(L_{i+j}\right) \cap O I^{k}\left(N_{i}^{\prime}\right)\right)
\end{gathered}
$$

for all $k \in \mathbf{N}$ and $i \in \mathbf{Z}$.

Note that $\left(A_{i}^{k+1}, B_{i}^{k+1}\right) \subseteq\left(A_{i}^{k}, B_{i}^{k}\right)$ for all $k \geq 0$ and $i \in \mathbf{Z}$ and there exists $m$ such that $A_{i}^{m}=N_{i}$ for all $i \in \mathbf{Z}$.

One can check that $f\left(A_{i}^{k} \backslash B_{i}^{k}\right) \subseteq A_{i}^{k+1} \cup f\left(A_{i}^{k+1}\right)$, thus each pair $\left\{\left(A_{i}^{k+1}, B_{i}^{k+1}\right)_{i}\right.$, $\left.\left(A_{i}^{k}, B_{i}^{k}\right)_{i}\right\}$ is as in Step 1 and consequently,

$$
\operatorname{Con}^{*}\left(\left(A_{i}^{k+1}, B_{i}^{k+1}\right)_{i}\right) \cong \operatorname{Con}^{*}\left(\left(A_{i}^{k}, B_{i}^{k}\right)_{i}\right) \text {, for all } k .
$$

Moreover, $\left\{\left(N_{i}, L_{i}\right)_{i},\left(A_{i}^{m}, B_{i}^{m}\right)_{i}\right\}$ and $\left\{\left(N_{i}^{\prime}, L_{i}^{\prime}\right)_{i},\left(A_{i}^{0}, B_{i}^{0}\right)_{i}\right\}$ are also as in Step 1, so we conclude that

$$
\operatorname{Con}^{*}\left(\left(N_{i}, L_{i}\right)_{i}\right) \cong \operatorname{Con}^{*}\left(\left(N_{i}^{\prime}, L_{i}^{\prime}\right)_{i}\right)
$$

Step 3. Assume that the sequences $\left(N_{i}, L_{i}\right)_{i}$ and $\left(N_{i}^{\prime}, L_{i}^{\prime}\right)_{i}$ satisfy only (2.7). Define $\left(N_{i}^{1}, L_{i}^{1}\right)_{i}:=\left(N_{i}, N_{i} \cap L_{i}^{\prime}\right)_{i}$ and $\left(N_{i}^{2}, L_{i}^{2}\right)_{i}:=\left(N_{i} \cup L_{i}^{\prime}, L_{i}^{\prime}\right)_{i}$. One can check that both $\left(N_{i}^{1}, L_{i}^{1}\right)_{i}$ and $\left(N_{i}^{2}, L_{i}^{2}\right)_{i}$ are index pair sequences. 
We have the following commutative diagram of inclusions:

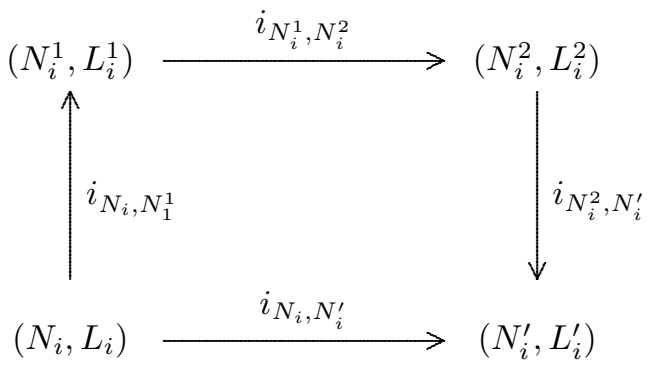

Note that $N_{i}^{1} \backslash L_{i}^{1}=N_{i}^{2} \backslash L_{i}^{2}=N_{i} \backslash L_{i}^{\prime}$ for all $i$, so $i_{N_{i}^{1}, N_{i}^{2}}$ induces an isomorphism for the cohomologies

$$
i_{N_{i}^{1}, N_{i}^{2}}^{*}: H^{*}\left(N_{i}^{2}, L_{i}^{2}\right) \rightarrow H^{*}\left(N_{i}^{1}, L_{i}^{1}\right)
$$

The pair of index pair sequences $\left\{\left(N_{i}, L_{i}\right)_{i},\left(N_{i}^{1}, L_{i}^{1}\right)_{i}\right\}$ satisfies the conditions $(2.7)$ and (2.9) and the pair $\left\{\left(N_{i}^{2}, L_{i}^{2}\right)_{i},\left(N_{i}^{\prime}, L_{i}^{\prime}\right)_{i}\right\}$ satisfies $(2.7)$ and (2.10).

Applying the Leray functor to the following diagram,

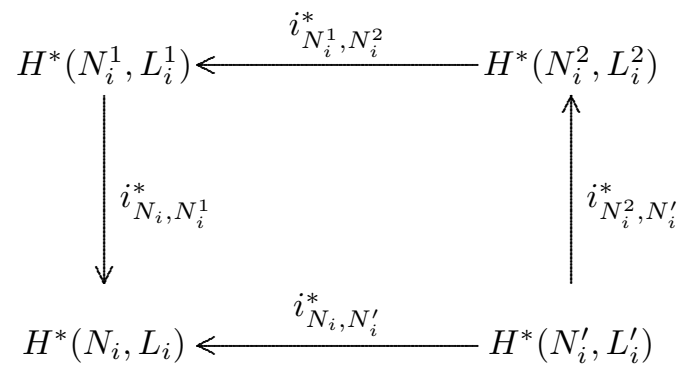

by Step 1 and Step 2 we conclude that

$$
\operatorname{Con}^{*}\left(\left(N_{i}, L_{i}\right)_{i}\right) \cong \operatorname{Con}^{*}\left(\left(N_{i}^{\prime}, L_{i}^{\prime}\right)_{i}\right) .
$$

Step 4. Suppose that $\left(N_{i}, L_{i}\right)_{i}$ is an index pair for $\left\{V_{i}\right\}_{i} \in\left[\left\{V_{i}\right\}_{i}\right]$. There exists $n>0$ such that $O I^{n}\left(V_{i}\right) \subseteq \operatorname{int}\left(N_{i} \backslash L_{i}\right) \subseteq \operatorname{cl}\left(N_{i} \backslash L_{i}\right) \subseteq \operatorname{int} V_{i} \subseteq V_{i}$ for all $i \in \mathbf{Z}$.

Let $\left(N_{i}^{\#}, L_{i}^{\#}\right)_{i}$ be the index pair sequence defined by:

$$
\begin{gathered}
N_{i}^{\#}=O I^{n}\left(V_{i}\right) \\
L_{i}^{\#}=O L^{n}\left(V_{i}\right):=\left\{x \in N_{i}^{\#}: \exists k \in\{0,1, \ldots, n+1\} \text { with } f^{k}(x) \notin \operatorname{int} V_{i+k}\right\} .
\end{gathered}
$$

Now we define the index pair sequence $\left(N_{i}^{b}, L_{i}^{b}\right)_{i}$ by:

$$
N_{i}^{b}:=N_{i} \cap \bigcap_{-n \leq k \leq 0} f^{-k}\left(V_{i+k}\right)
$$

and

$$
L_{i}^{b}:=\left\{x \in N_{i}^{b}: \exists k \in\{0,1, \ldots, n+1\} \text { with } f^{k}(x) \notin V_{i+k}\right\}
$$

and the index pair sequence $\left(N_{i}^{b}, N_{i}^{b} \cap L_{i}\right)_{i}$.

We will have the following inclusions of index pair sequences:

$$
\begin{aligned}
& \left(N_{i}^{b}, N_{i}^{b} \cap L_{i}\right)_{i} \subseteq\left(N_{i}, L_{i}\right)_{i} \\
& \left(N_{i}^{b}, L_{i}^{b}\right)_{i} \subseteq\left(N_{i}^{b}, N_{i}^{b} \cap L_{i}\right)_{i}
\end{aligned}
$$




$$
\left(N_{i}^{\#}, L_{i}^{\#}\right)_{i} \subseteq\left(N_{i}^{b}, L_{i}^{b}\right)_{i}
$$

Applying Step 3 for each of them, we finally conclude that

$$
\mathcal{L}\left(\left(H^{*}\left(N_{i}, L_{i}\right), F_{N_{i}}^{*}\right)_{i}\right)=\mathcal{L}\left(\left(H^{*}\left(N_{i}^{\#}, L_{i}^{\#}\right), F_{N_{i}^{\#}}^{*}\right)_{i}\right) .
$$

Step 5. Suppose that the index pair sequences $\left(N_{i}, L_{i}\right)_{i}$ for $\left\{V_{i}\right\}_{i} \in\left[\left\{V_{i}\right\}_{i}\right]$ and $\left(N_{i}^{\prime}, L_{i}^{\prime}\right)_{i}$ for $\left\{V_{i}^{\prime}\right\}_{i} \in\left[\left\{V_{i}^{\prime}\right\}_{i}\right]$, respectively, do not satisfy any extra assumptions. By Step 4 we have that

$$
\operatorname{Con}^{*}\left(\left(N_{i}, L_{i}\right)_{i}\right) \simeq \operatorname{Con}^{*}\left(\left(O I^{n_{1}}\left(V_{i}\right), O L^{n_{1}}\left(V_{i}\right)\right)\right.
$$

if $n_{1}$ is large enough and

$$
\operatorname{Con}^{*}\left(\left(N_{i}^{\prime}, L_{i}^{\prime}\right)_{i}\right) \simeq \operatorname{Con}^{*}\left(\left(O I^{n_{2}}\left(V_{i}^{\prime}\right), O L^{n_{2}}\left(V_{i}^{\prime}\right)\right)\right.
$$

if $n_{2}$ is large enough.

On the other hand, $O I^{m}\left(V_{i}\right) \subseteq \operatorname{int}\left(V_{i}^{\prime}\right)$ for $m$ large and so $\left(\left(O I^{m}\left(V_{i}\right), O L^{m}\left(V_{i}\right)\right)_{i}\right.$ is an index pair sequence for $\left\{V_{i}^{\prime}\right\}_{i}$. This implies that

$$
\operatorname{Con}^{*}\left(( O I ^ { n _ { 1 } } ( V _ { i } ) , O L ^ { n _ { 1 } } ( V _ { i } ) ) \simeq \operatorname { C o n } ^ { * } \left(\left(O I^{n_{2}}\left(V_{i}^{\prime}\right), O L^{n_{2}}\left(V_{i}^{\prime}\right)\right)\right.\right.
$$

if $n_{1}, n_{2}$ are large enough. Thus

$$
\operatorname{Con}^{*}\left(\left(N_{i}, L_{i}\right)_{i}\right) \simeq \operatorname{Con}^{*}\left(\left(N_{i}^{\prime}, L_{i}^{\prime}\right)_{i}\right) .
$$

This ends the proof of the theorem.

EXAmple 2.9. We return to the horseshoe and the billiard system in Example 2.5 and Example 2.6, respectively . For both of the examples, for all $\left(a_{i}\right)_{i}$ and $i \in \mathbf{Z}$ we compute:

$$
H^{k}\left(N_{a_{i}}, L_{a_{i}}\right)= \begin{cases}\mathbf{Z} & \text { for } k=1 \\ 0 & \text { otherwise }\end{cases}
$$

The system (2.4) at level 1 becomes:

$$
\ldots \rightarrow \mathbf{Z} \stackrel{ \pm i d}{\longrightarrow} \mathbf{Z} \stackrel{ \pm i d}{\longrightarrow} \mathbf{Z} \stackrel{ \pm i d}{\longrightarrow} \mathbf{Z} \rightarrow \ldots
$$

and is the zero object of $\mathcal{S}$ at every other level. The system (2.14) already represents a graded directed system of modules and isomorphisms so it stays the same after applying the functor $\mathcal{L}$. Thus the Conley index $\operatorname{Con}^{*}\left[\left\{V_{a_{i}}\right\}_{i}\right]$ of $\left[\left\{V_{a_{i}}\right\}_{i}\right]$ is zero at every level $k \neq 1$ and at level $k=1$ takes the form of the system (2.18) or of the pair $(\mathbf{Z}, \pm i d)$.

3. Properties of the index. We will show that the fundamental properties satisfied by the Conley index in general still hold for this index.

THEOREM 3.1 (Ważewski property). If $C_{0}{ }^{*}\left(\left\{V_{i}\right\}_{i}\right)$ is not isomorphic to 0 , then there exists a point $x \in V_{0}$ with $f^{i}(x) \in V_{i}$ for all $i \in \mathbf{Z}$, i.e. $O I\left(V_{0}\right) \neq \emptyset$.

Proof. Suppose by contradiction that $O I\left(V_{0}\right)=\emptyset$. By the finite intersection property, there exists $n>0$ such that $O I^{n}\left(V_{0}\right)=\emptyset$. There also exists $m>n$ such that $O I^{m}\left(V_{i}\right) \subseteq$ $i n t V_{i}$ for all $i \in \mathbf{Z}$. As in the proof of Theorem 2.4, we can obtain $\left(N_{i}, L_{i}\right)_{i \in \mathbf{Z}}$ an index pair sequence for $\left\{V_{i}\right\}_{i}$ with $N_{i}=O I^{m}\left(V_{i}\right)$. It implies that $\left(N_{0}, L_{0}\right)=(\emptyset, \emptyset)$. Thus the directed system of $R$-modules and homomorphisms (2.4) becomes:

$$
\ldots H^{*}\left(N_{2}, L_{2}\right) \stackrel{F_{N_{1}}^{*}}{\longrightarrow} H^{*}\left(N_{1}, L_{1}\right) \stackrel{F_{N_{0}}^{*}}{\longrightarrow} 0 \stackrel{0}{\longrightarrow} H^{*}\left(N_{-1}, L_{-1}\right) \stackrel{F_{N-2}^{*}}{\longrightarrow} H^{*}\left(N_{-2}, L_{-2}\right) \ldots
$$


Applying $\mathcal{L}$ to this graded directed system of modules and homomorphism we obtain:

$$
\ldots \rightarrow 0 \stackrel{0}{\longrightarrow} 0 \stackrel{0}{\longrightarrow} 0 \stackrel{0}{\longrightarrow} 0 \rightarrow \ldots
$$

contradiction to our hypothesis.

THEOREM 3.2 (Summation property). Assume that $\left\{V_{i}\right\}_{i},\left\{V_{i}^{\prime}\right\}_{i} \in \mathcal{W}$, thus there exists $n, n^{\prime}>0$, such that $O I^{n}\left(V_{i}\right) \subseteq$ int $V_{i}$ and $O I^{n^{\prime}}\left(V_{i}^{\prime}\right) \subseteq$ int $V_{i}^{\prime}$ for all $i \in \mathbf{Z}$. Suppose that $V_{i} \cap V_{i}^{\prime}=\emptyset$ and $O I^{m}\left(V_{i} \cup V_{i}^{\prime}\right)=O I^{m}\left(V_{i}\right) \cup O I^{m}\left(V_{i}^{\prime}\right)$ for some $m \geq n, n^{\prime}$ and all $i \in \mathbf{Z}$. Then $\left\{V_{i} \cup V_{i}^{\prime}\right\}_{i} \in \mathcal{W}$ and $\operatorname{Con}^{*}\left(\left\{V_{i} \cup V_{i}^{\prime}\right\}_{i}\right) \cong \operatorname{Con}^{*}\left(\left\{V_{i}\right\}_{i}\right) \oplus \operatorname{Con}^{*}\left(\left\{V_{i}^{\prime}\right\}_{i}\right)$.

Proof. $O I^{m}\left(V_{i} \cup V_{i}^{\prime}\right)=O I^{m}\left(V_{i}\right) \cup O I^{m}\left(V_{i}^{\prime}\right) \subseteq \operatorname{int}\left(V_{i}\right) \cup \operatorname{int}\left(V_{i}^{\prime}\right) \subseteq \operatorname{int}\left(V_{i} \cup V_{i}^{\prime}\right)$ for all $i \in \mathbf{Z}$ shows that $\left\{V_{i} \cup V_{i}^{\prime}\right\}_{i} \in \mathcal{W}$. Let $\left(N_{i}, L_{i}\right)_{i}$ be an index pair sequence for $\left\{O I^{1}\left(V_{i}\right)\right\}_{i} \sim\left\{V_{i}\right\}_{i}$ and $\left(N_{i}^{\prime}, L_{i}^{\prime}\right)_{i}$ an index pair sequence for $\left\{O I^{1}\left(V_{i}^{\prime}\right)\right\}_{i} \sim\left\{V_{i}^{\prime}\right\}_{i}$. One can prove that $\left(\widehat{N}_{i}, \widehat{L}_{i}\right)_{i}=\left(N_{i} \cup N_{i}^{\prime}, L_{i} \cup L_{i}^{\prime}\right)_{i}$ is an index pair sequence for $\left\{V_{i} \cup V_{i}^{\prime}\right\}_{i}$. Since $H^{*}\left(\widehat{N}_{i}, \widehat{L}_{i}\right)=H^{*}\left(N_{i}, L_{i}\right) \oplus H^{*}\left(N_{i}^{\prime}, L_{i}^{\prime}\right)$ and $F_{\widehat{N}_{i}}^{*}=F_{N_{i}}^{*} \oplus F_{N_{i}^{\prime}}^{*}$, when we apply the Leray functor (see Proposition 2.2.3 in [Gi3]) we obtain

$$
\mathcal{L}\left(\left(H^{*}\left(\widehat{N}_{i}, \widehat{L}_{i}\right), F_{N_{i}}^{*}\right)_{i}\right)=\mathcal{L}\left(\left(H^{*}\left(N_{i}, L_{i}\right), F_{N_{i}}^{*}\right)_{i}\right) \oplus \mathcal{L}\left(\left(H^{*}\left(N_{i}^{\prime}, L_{i}^{\prime}\right), F_{N_{i}^{\prime}}^{*}\right)_{i}\right)
$$

which concludes our proof.

The continuation property holds under special conditions on the nature of the index pair sequence $\left\{V_{i}\right\}_{i}$. If $\delta>0$ and $A$ is a closed subset of $X, A(\delta)$ will denote the closed $\delta$-neighborhood of $A$ defined by

$$
A(\delta)=\bigcup_{x \in A} \bar{B}(x, \delta)
$$

where $\bar{B}(x, \delta)=\{y \in X \mid d(x, y) \leq \delta\}$. Note that if $A$ is closed the $A(\delta)$ is also closed.

Denote:

$$
\begin{aligned}
& \mathcal{W}_{0}=\mathcal{W}_{0}(f):=\left\{\left\{V_{i}\right\}_{i \in \mathbf{Z}} \mid V_{i} \text { is compact, } \bigcup_{i} V_{i}\right. \text { is relatively compact } \\
& \\
& \text { and there exists } \delta_{1}, \delta_{2}>0 \text { and } m \in \mathbf{N} \text { such that } \\
&\left.O I_{f}^{m}\left(V_{i}\left(\delta_{1}\right)\right)\left(\delta_{2}\right) \subset i n t V_{i} \text { for all } i \in \mathbf{Z}\right\} .
\end{aligned}
$$

Here $O I_{f}^{m}\left(V_{i}\left(\delta_{1}\right)\right)$ means $\left\{x \in X \mid f^{i+j}(x) \in V_{i+j}\left(\delta_{1}\right)\right.$ for all $\left.j \in\{-m, \ldots, m\}\right\}$. It is clear that if $\left\{V_{i}\right\}_{i} \in \mathcal{W}_{0}$ then $\left\{V_{i}\left(\delta_{1}\right)\right\}_{i} \in \mathcal{W}_{0}$ and $\left\{V_{i}\right\}_{i} \sim\left\{V_{i}\left(\delta_{1}\right)\right\}_{i}$ for $\delta_{1}>0$ small enough.

Let $\Lambda$ be a compact interval in $\mathbf{R}$ and $f: \Lambda \times X \rightarrow \Lambda \times X$ a homeomorphism which is parameter preserving, i.e. $f(\{\lambda\} \times X) \subseteq\{\lambda\} \times X$ for each $\lambda \in \Lambda$. The map $f_{\lambda}: X \rightarrow X$ is defined by $f(\lambda, x)=\left(\lambda, f_{\lambda}(x)\right)$ for each $\lambda \in \Lambda$ and it is a homeomorphism of $X$.

LEMmA 3.3. If $\left\{V_{i}\right\}_{i} \in \mathcal{W}_{0}\left(f_{\mu}\right)$ for some $\mu \in \Lambda$, then there exists $\eta>0$ such that $\left\{V_{i}\right\}_{i} \in \mathcal{W}_{0}\left(f_{\lambda}\right)$ for all $\lambda \in(\mu-\eta, \mu+\eta)$.

Proof. Let $\delta_{1}, \delta_{2}>0$ and $m \in \mathbf{N}$ such that $O I_{f_{\mu}}^{m}\left(V_{i}\left(2 \delta_{1}\right)\right)\left(\delta_{2}\right) \subseteq i n t V_{i}$ for all $i \in \mathbf{Z}$ and $\operatorname{cl}\left(\bigcup_{i} V_{i}\left(\delta_{1}\right)\right)$ is compact. Since $f$ is uniformly continuous on $\Lambda \times \operatorname{cl}\left(\bigcup_{i} V_{i}\left(2 \delta_{1}\right)\right)$ then, there exists $\eta>0$ (with $\eta<\delta_{2}$ ) such that for all $\lambda \in \Lambda$ with $|\lambda-\mu|<\eta$ and all $x, y \in \operatorname{cl}\left(\bigcup_{i} V_{i}\left(2 \delta_{1}\right)\right)$ with $d(x, y)<\eta$ it follows that $d\left(f_{\lambda}^{n}(x), f_{\mu}^{n}(y)\right)<\delta_{1}$ for all $n \in\{-m, \ldots, m\}$.

If $x \in O I_{f_{\lambda}}^{m}\left(V_{i}\left(\delta_{1}\right)\right)(\eta)$ for some $i \in \mathbf{Z}$, then there exists $y \in O I_{f_{\lambda}}^{m}\left(V_{i}\left(\delta_{1}\right)\right)$ with $d(x, y)<\eta$, hence $f_{\lambda}^{n}(y) \in V_{i+n}\left(\delta_{1}\right)$ for all $n \in\{-m, \ldots, m\}$, so $f_{\mu}^{n}(x) \in V_{i+n}\left(2 \delta_{1}\right)$ for all 
$n \in\{-m, \ldots, m\}$, thus $x \in O I_{f_{\mu}}^{m}\left(V_{i}\left(2 \delta_{1}\right)\right) \subseteq \operatorname{int} V_{i}$. In conclusion $O I_{f_{\lambda}}^{m}\left(V_{i}\left(\delta_{1}\right)\right)(\eta) \subseteq \operatorname{int} V_{i}$ for all $i \in \mathbf{Z}$ and $\lambda \in(\mu-\eta, \mu+\eta)$.

THEOREM 3.4. If $\left\{f_{\lambda}\right\}_{\lambda}$ is as above and there exists $\left\{V_{i}\right\}_{i}$ an isolating neighborhood sequence in $\mathcal{W}_{0}\left(f_{\lambda}\right)$ for all $\lambda \in \Lambda$, then $\operatorname{Con}^{*}\left(\left\{V_{i}\right\}_{i}, f_{\lambda}\right)$ does not depend on $\lambda \in \Lambda$.

Proof. Let $\delta_{1}, \delta_{2}>0$ and $n \in \mathbf{N}$ such that $O I^{n}\left(V_{i}\left(3 \delta_{1}\right)\right)\left(\delta_{2}\right) \subseteq \operatorname{int} V_{i}$ for all $i \in \mathbf{Z}$. We can assume that $\delta_{1}=\delta_{2}$. Since $f$ is uniformly continuous on $\Lambda \times \operatorname{cl}\left(\bigcup_{i} V_{i}\left(3 \delta_{1}\right)\right)$,

$$
\text { there exists } \left.\delta>0 \text { (with } \delta<\delta_{1}\right) \text { such that if } x, y \in c l\left(\bigcup_{i} V_{i}\left(3 \delta_{1}\right)\right) \text { with } d(x, y)<\delta \text {, }
$$
then $d\left(f_{\mu}^{k}(x), f_{\mu}^{k}(y)\right)<\delta_{1}$ for all $k \in\{-8 n, \ldots, 8 n\}$.

Applying the uniform continuity of $f$ on $\Lambda \times \operatorname{cl}\left(\bigcup_{i} V_{i}\left(3 \delta_{1}\right)\right)$ one more time we obtain that:

(3.4) there exists $\eta>0$ such that if $|\lambda-\mu|<\eta$ and $x \in \operatorname{cl}\left(\bigcup_{i}\left(V_{i}\left(3 \delta_{1}\right) z\right)\right)$ then $d\left(f_{\lambda}^{k}(x), f_{\mu}^{k}(x)\right)<\delta$ for all $k \in\{-8 n, \ldots, 8 n\}$.

For convenience, we will fix $\mu \in \Lambda$ and denote $f_{\mu}$ by $f$ from now on. Define:

$$
\begin{gathered}
N_{i}:=O I^{n}\left(V_{i}\right), \\
L_{i}:=O L^{n}\left(V_{i}\right)=\left\{x \in N_{i} \mid \exists k \in\{0, \ldots, n+1\} \text { such that } f^{k}(x) \notin i n t V_{i+k}\right\}, \\
N_{i}^{1}:=O I^{5 n}\left(V_{i}\right), \\
L_{i}^{1}:=O L^{5 n}\left(V_{i}\right)=\left\{x \in N_{i}^{1} \mid \exists k \in\{0, \ldots, 5 n+1\} \text { such that } f^{k}(x) \notin i n t V_{i+k}\right\}, \\
N_{i}^{2}:=N_{i}, \\
L_{i}^{2}:=\left\{x \in N_{i} \mid \exists k \in\{0, \ldots, 7 n+1\} \text { such that } f^{k}(x) \notin V_{i+k}\right\},
\end{gathered}
$$

for all $i \in \mathbf{Z}$.

We claim that for every $\lambda \in(\mu-\eta, \mu+\eta)$ there exists an index pair sequence $\left(N_{i}^{\lambda}, L_{i}^{\lambda}\right)_{i}$ for $\left\{V_{i}\right\}_{i}$ with respect to $f_{\lambda}$ such that:

$$
\left(N_{i}^{1}, L_{i}^{1}\right)_{i} \subseteq\left(N_{i}^{\lambda}, L_{i}^{\lambda}\right)_{i} \subseteq\left(N_{i}^{2}, L_{i}^{2}\right)_{i}
$$

and the inclusions

$$
i_{i}:\left(N_{i}^{1}, L_{i}^{1}\right)_{i} \rightarrow\left(N_{i}^{\lambda}, L_{i}^{\lambda}\right)_{i} \text { and } j_{i}:\left(N_{i}^{\lambda}, L_{i}^{\lambda}\right)_{i} \rightarrow\left(N_{i}^{2}, L_{i}^{2}\right)_{i}
$$

induce morphisms between objects of $\mathcal{S}$

$$
\begin{aligned}
& i_{i}^{*}:\left(H^{*}\left(N_{i}^{\lambda}, L_{i}^{\lambda}\right), F_{N_{i}^{\lambda}}^{*}\right)_{i} \rightarrow\left(H^{*}\left(N_{i}^{1}, L_{i}^{1}\right), F_{N_{i}^{1}}^{*}\right)_{i} \\
& j_{i}^{*}:\left(H^{*}\left(N_{i}^{2}, L_{i}^{2}\right), F_{N_{i}^{2}}^{*}\right)_{i} \rightarrow\left(H^{*}\left(N_{i}^{\lambda}, L_{i}^{\lambda}\right), F_{N_{i}^{\lambda}}^{*}\right)_{i}
\end{aligned}
$$

Define

$$
\begin{gathered}
N_{i}^{\lambda}=\operatorname{cl}\left(N_{i} \backslash L_{i}\right) \cap \bigcap_{-4 n \leq k \leq 0} f_{\lambda}^{-k}\left(V_{i+k}\left(\delta_{1}\right)\right) \\
L_{i}^{\lambda}=N_{i}^{\lambda} \cap\left\{x \in N_{i}^{\lambda} \mid \exists k \in\{0, \ldots, 6 n+1\} \text { such that } f^{k}(x) \notin i n t V_{i+k}\right\}
\end{gathered}
$$

for each $i \in \mathbf{Z}$ and each $\lambda \in(\mu-\eta, \mu+\eta)$. The fact that $\left(N_{i}^{1}, L_{i}^{1}\right)_{i}$ and $\left(N_{i}^{2}, L_{i}^{2}\right)_{i}$ are index pair sequences for $\left\{V_{i}\right\}_{i}$ with respect to $f$ satisfying $\left(N_{i}^{1}, L_{i}^{1}\right)_{i} \subset\left(N_{i}^{2}, L_{i}^{2}\right)_{i}$ and $\left(N_{i}^{\lambda}, L_{i}^{\lambda}\right)_{i}$ 
is an index pair sequence for $\left\{V_{i}\right\}_{i}$ with respect to $f_{\lambda}$ follow similarly. It remains to prove $(3.5)$.

If $x \in N_{i}^{1}$ then $f^{k}(x) \in V_{i+k}$ for all $k \in\{-5 n, \ldots, 5 n\}$ in particular $x \in O I^{2 n+1}\left(V_{i}\right) \subseteq$ $\operatorname{cl}\left(N_{i} \backslash L_{i}\right)$ and $f_{\lambda}^{k}(x) \in V_{i+k}\left(\delta_{1}\right)$ for all $k \in\{-4 n, \ldots, 0\}$ thus $x \in \operatorname{cl}\left(N_{i} \backslash L_{i}\right) \cap$ $\bigcap_{-4 n<k<0} f_{\lambda}^{-k}\left(V_{i+k}\left(V\left(\delta_{1}\right)\right)\right)=N_{i}^{\lambda}$. Suppose that $x \in L_{i}^{\lambda}$ and $x \in N_{i}^{1} \backslash L_{i}^{\lambda}$ so $x \in$ $N_{i}^{1} \cap\left(N^{\lambda} \backslash L_{i}^{\lambda}\right)$, consequently

$$
x \in \bigcap_{-4 n \leq k \leq 0} f_{\lambda}^{-k}\left(V_{i+k}\left(V\left(\delta_{1}\right)\right)\right) \cap \bigcap_{0 \leq k \leq 6 n+1} f_{\lambda}^{-k}\left(i n t V_{i+k}\left(V\left(\delta_{1}\right)\right)\right),
$$

in particular $f_{\lambda}^{k}(x) \in V_{i+k}\left(\delta_{1}\right)$ for all $k \in\{-4 n, \ldots, 6 n+1\}$, hence $f^{k}(x) \in O I^{n}\left(V_{i+k}\left(\delta_{1}\right)\right)$ $\subseteq i n t V_{i+k}$ for all $k \in\{-3 n, \ldots, 5 n+1\}$, thus $f^{k}(x) \in i n t V_{i+k}$ for all $0 \leq k \leq 5 n+1$, concluding that $x \in N_{i}^{1} \backslash L_{i}^{1}$, contradiction. We have checked that $\left(N_{i}^{1}, L_{i}^{1}\right)_{i} \subseteq\left(N_{i}^{\lambda}, L_{i}^{\lambda}\right)_{i}$. If $x \in N_{i}^{\lambda}$, then $x \in \operatorname{cl}\left(N_{i} \backslash L_{i}\right) \subset N_{i}^{2}$. If $x \in L_{i}^{\lambda}$, then $x \in N_{i}^{\lambda} \subseteq N_{i}^{2}$ and there exists $j \in\{0, \ldots, 6 n+1\}$ with $f_{\lambda}^{j}(x) \notin \operatorname{int}\left(V_{i+j}\left(\delta_{1}\right)\right)$ thus $f^{j}(x) \notin \operatorname{int} V_{i+j}$ for some $j \in\{0, \ldots, 6 n+1\} \subseteq\{0, \ldots, 7 n+1\}$. We have checked that $\left(N_{i}^{\lambda}, L_{i}^{\lambda}\right)_{i} \subseteq\left(N_{i}^{2}, L_{i}^{2}\right)_{i}$, which ends the proof of (3.5).

Now we would like to prove (3.6) and (3.7). In order to do this, fix $\kappa \in(\mu-\eta, \mu+\eta)$ and prove that for all $\lambda \in(\mu-\eta, \mu+\eta)$, the following inclusions hold:

$$
\begin{aligned}
f_{\lambda}\left(N_{i}^{1}, L_{i}^{1}\right) \subseteq\left(N_{i+1}^{\kappa} \cup f_{\kappa}\left(N_{i}^{\kappa}\right), L_{i+1}^{\kappa} \cup f_{\kappa}\left(L_{i}^{\kappa}\right)\right) \\
f_{\lambda}\left(N_{i}^{\kappa}, L_{i}^{\kappa}\right) \subseteq\left(N_{i+1}^{2} \cup f\left(N_{i}^{2}\right), L_{i+1}^{2} \cup f\left(L_{i}^{2}\right)\right)
\end{aligned}
$$

It is enough to prove that $f_{\lambda}\left(N_{i}^{1}\right) \subset f_{\kappa}\left(N_{i}^{\kappa}\right), f_{\lambda}\left(L_{i}^{1}\right) \subset f_{\kappa}\left(L_{i}^{\kappa}\right), f_{\lambda}\left(N_{i}^{1}\right) \subset f\left(N_{i}^{2}\right)$ and $f_{\lambda}\left(L_{i}^{\kappa}\right) \subset f\left(L_{i}^{2}\right)$ for all $i \in \mathbf{Z}$.

- $f_{\lambda}\left(N_{i}^{1}\right) \subset f_{\kappa}\left(N_{i}^{\kappa}\right) \Longleftrightarrow f_{\kappa}^{-1} f_{\lambda}\left(N_{i}^{1}\right) \subseteq N_{i}^{\kappa}$. If $y \in f_{\kappa}^{-1} f_{\lambda}\left(N_{i}^{1}\right)$, then $f_{\lambda}^{-1} f_{\kappa}(y) \in N_{i}^{1}$, so $y=f_{\kappa}^{-1} f_{\kappa}(y) \in N_{i}^{1}(\delta)=O I_{f}^{5 n}\left(V_{i}\right)(\delta)$.

We claim that $O I_{f}^{5 n}\left(V_{i}\right)(\delta) \subseteq O I^{4 n}\left(V_{i}\right)$. Indeed, if $x \in O I_{f}^{5 n}\left(V_{i}\right)(\delta)$, then there exists $z$ with $d(x, z)<\delta$ and $z \in O I_{f}^{5 n}\left(V_{i}\right)$, hence $f^{k}(z) \in V_{i+k}$ for all $k \in\{-5 n, \ldots, 5 n\}$ so $f^{k}(z) \in O I^{n}\left(V_{i+k}\right)$ for all $k \in\{-4 n, \ldots, 4 n\}$, thus $f^{k}(x) \in O I^{n}\left(V_{i+k}\right)\left(\delta_{1}\right) \subseteq V_{i+k}$ for all $k \in\{-4 n, \ldots, 4 n\}$, in other words $x \in O I^{4 n}\left(V_{i}\right)$, ending the proof of the above claim.

Thus $y \in O I^{4 n}\left(V_{i}\right) \subseteq \operatorname{cl}\left(N_{i} \backslash L_{i}\right) \cap \bigcap_{-4 n \leq k \leq 0} f_{\kappa}^{-k}\left(V_{i+k}\left(\delta_{1}\right)\right)=N_{i}^{\kappa}$

- $f_{\lambda}\left(L_{i}^{1}\right) \subset f_{\kappa}\left(L_{i}^{\kappa}\right) \Longleftrightarrow f_{\kappa}^{-1} f_{\lambda}\left(L_{i}^{1}\right) \subseteq L_{i}^{\kappa}$.

If $y \in f_{\kappa}^{-1} f_{\lambda}\left(L_{i}^{1}\right)$, then $f_{\lambda}^{-1} f_{\kappa}(y) \in L_{i}^{1}$, so $y=f_{\kappa}^{-1} f_{\kappa}(y) \in L_{i}^{1}(\delta)$.

We claim that $L_{i}^{1}(\delta) \subset L_{1}^{\kappa}$, which will prove our inclusion. Since $N_{i}^{1}(\delta) \subset N_{i}^{\kappa}$, it is enough to prove that $\left(N_{i}^{1} \backslash L_{i}^{\kappa}\right)(\delta) \cap N_{i}^{1} \subseteq N_{i}^{1} \backslash L_{i}^{1}$. Take $x \in\left(N_{i}^{1} \backslash L_{i}^{\kappa}\right)(\delta) \cap N_{i}^{1} \subset$ $N_{i}^{1} \cap\left(N_{i}^{1} \cap \bigcap_{0 \leq k \leq 6 n+1} f_{\kappa}^{-k}\left(V_{i+k}\left(\delta_{1}\right)\right)(\delta)\right.$. There exists $z \in \bigcap_{-n \leq k \leq 6 n+1} f_{\kappa}^{-k}\left(V_{i+k}\left(\delta_{1}\right)\right)$ with $d(x, z)<\delta$, hence $f_{\kappa}^{k}(z) \in V_{i+k}\left(\delta_{1}\right)$ for all $k \in\{-n, \ldots, 6 n+1\}$, so $f^{k}(z) \in$ $V_{i+k}\left(2 \delta_{1}\right)$ for all $k \in\{-n, \ldots, 6 n+1\}$, thus $f^{k}(x) \in O I^{n}\left(V_{i+k}\right)\left(3 \delta_{1}\right) \subseteq i n t V_{i+k}$ for all $k \in\{0, \ldots, 5 n+1\}$. We obtain that $x \in N_{i}^{1} \cap \bigcap_{0 \leq k \leq 5 n+1} f^{-k}\left(\right.$ int $\left.V_{i+k}\right)=N_{i}^{1} \backslash L_{i}^{1}$. This ends the proof of the claim.

The other two inclusions follow similarly.

We obtain a homotopy commutative diagram with vertical rows inclusions 


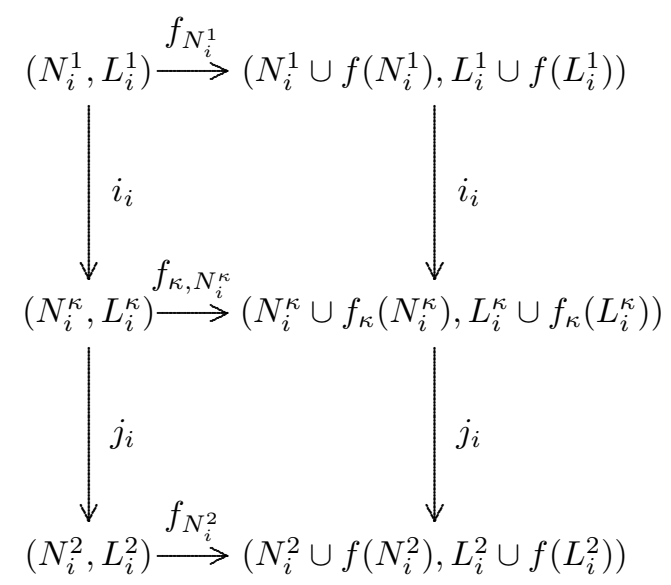

for every $i \in \mathbf{Z}$.

Since $f_{\kappa}$ and $f$ are homotopic, applying the Alexander-Spanier cohomology functor to the above diagram and extending it to the right and to the left provides us with the following commutative diagram:

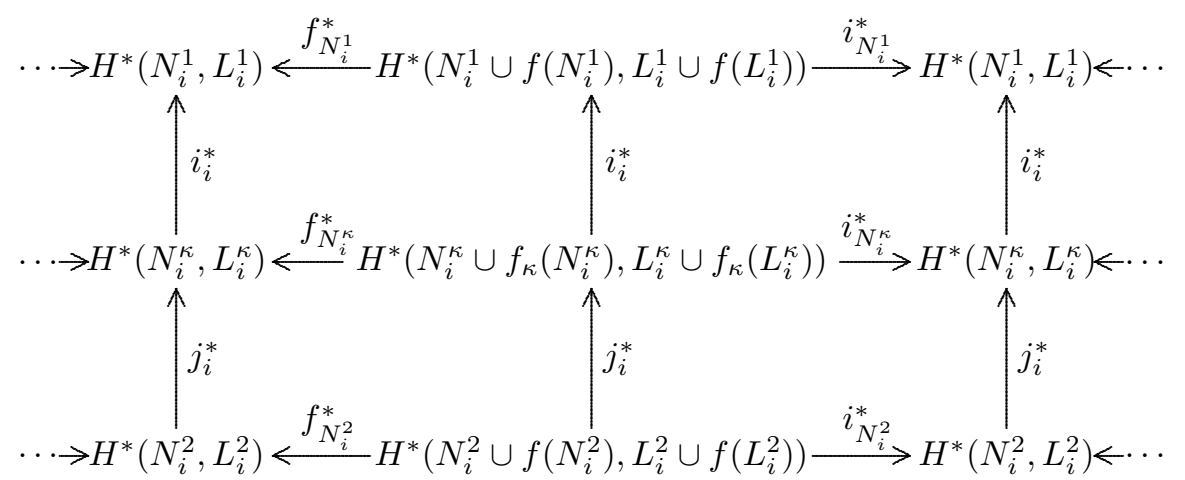

After removing $H^{*}\left(N_{i+1}^{1} \cup f\left(N_{i}^{1}\right), L_{i+1}^{1} \cup f\left(L_{i}^{1}\right)\right), H^{*}\left(N_{i+1}^{\kappa} \cup f_{\kappa}\left(N_{i}^{\kappa}\right), L_{i+1}^{\kappa} \cup f_{\kappa}\left(L_{i}^{\kappa}\right)\right)$, $H^{*}\left(N_{i+1}^{2} \cup f\left(N_{i}^{2}\right), L_{i+1}^{2} \cup f\left(L_{i}^{2}\right)\right)$ from the above diagram, we obtain a commutative diagram as below:

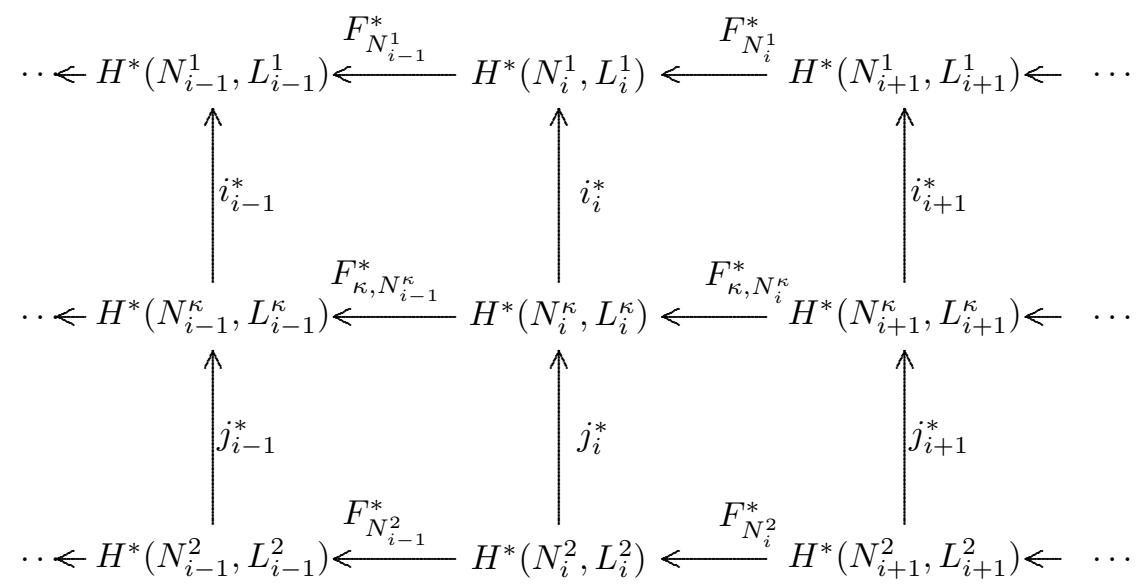


It follows that $\mathcal{L}\left(i_{i}^{*} \circ j_{i}^{*}\right)=\mathcal{L}\left(i_{i}^{*}\right) \circ \mathcal{L}\left(j_{i}^{*}\right)$ is an isomorphism, thus $\mathcal{L}\left(i_{i}^{*}\right)$ and $\mathcal{L}\left(j_{i}^{*}\right)$ are injective and surjective homomorphisms, respectively.

Since

$$
\mathcal{L}\left(\left(H^{*}\left(N_{i}^{1}, L_{i}^{1}\right), F_{N_{i}^{1}}^{*}\right)_{i}\right) \cong \mathcal{L}\left(\left(H^{*}\left(N_{i}^{2}, L_{i}^{2}\right), F_{N_{i}^{2}}^{*}\right)_{i}\right)
$$

we conclude that $\mathcal{L}\left(\left(H^{*}\left(N_{i}^{\kappa}, L_{i}^{\kappa}\right), F_{N_{i}^{\kappa}}^{*}\right)_{i}\right) \cong \mathcal{L}\left(\left(H^{*}\left(N_{i}^{1}, L_{i}^{1}\right), F_{N_{i}^{1}}^{*}\right)_{i}\right)$, for all $k \in(\mu-\eta$, $\mu+\eta)$. A compactness argument extends the above isomorphism to all of $\Lambda$.

4. Detection of periodic orbits and chaos. For the rest of the section all of the considered sets are ANR's. The following theorem is a version of Theorem 1 in [Sr] and an extension of Theorem 4 in [Mr2]. For the definitions and properties of the fixed point index and Lefschetz number, the reader is invited to see [Gr].

THEOREM 4.1. Let $\left(N_{i}, L_{i}\right)_{i}$ be an index pair sequence such that $\left(N_{i}, L_{i}\right)=\left(N_{i+k}, L_{i+k}\right)$ for some $k \in \mathbf{Z}^{+}$and all $i \in \mathbf{Z}$. If Lef $\left(F_{0}^{*} \circ F_{1}^{*} \circ \ldots F_{k-1}^{*}\right) \neq 0$ then there exists $x_{j} \in N_{j} \backslash L_{j}$ such that $f\left(x_{j}\right)=x_{j+k}$ for all $j \in\{0,1, \ldots, k-1\}$, so $f_{N_{k-1}} \circ f_{N_{k-2}} \ldots \circ f_{N_{0}}$ has a fixed point in $N_{0} \backslash L_{0}$. Thus the periodic orbit $\left\{x_{j}\right\}_{j}$ is a subset of $S$.

The next statement is also a version of Corollary 1 in [Sr] and partially extends Theorem 2.3 in [MiMr2] and Theorem 1.1 in [CaKwMi].Let $\kappa$ denote the ordinal number of $\mathcal{V}$ and let $\Sigma$ denote the space of all bi-infinite sequences of symbols in $\{1,2, \ldots, \kappa\}$ and $\sigma$ the shift map. Let $A=\left\{\alpha_{i j}\right\}_{i, j \in\{1,2, \ldots, \kappa\}}$ be a $\kappa \times \kappa$ (possible infinite) matrix with entries in $\{0,1\}$ and $\Sigma_{A}=\left\{a=\left(a_{i}\right)_{i \in \mathbf{Z}} \in \Sigma \mid \alpha_{a_{i} a_{i+1}}=1\right.$ for all $i$ in $\left.\mathbf{Z}\right\}$. Consider the restriction $\sigma_{A}$ of $\sigma$ on $\Sigma_{A}$.

Corollary 4.2. Let $\left\{N_{i}, L_{i}\right\}_{i \in\{1,2, \ldots, \kappa\}}$ be a sequence of pairwise disjoint (mod 0$)$ compact sets and suppose that $\left(N_{a_{i}}, L_{a_{i}}\right)_{i \in \mathbf{Z}}$ is an index pair sequence for every $a \in \Sigma_{A}$.

If $C H^{*}\left(\left(N_{a_{i}}, L_{a_{i}}\right)_{i}\right) \neq 0$ for all $a \in \Sigma_{A}$ then there exists $S \subseteq N_{1} \cup N_{2} \cup \ldots \cup N_{\kappa}$ an invariant set with respect to $f$ and a surjective map $\phi: S \rightarrow \Sigma_{A}$ such that the following diagram commutes:

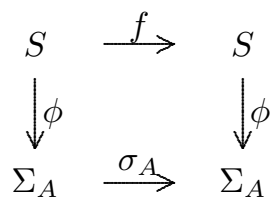

Moreover, if $C H^{m}\left(\left(N_{a_{i}}, L_{a_{i}}\right)_{i}\right)=(R$, isomorphism $)$ or $C H^{m}\left(\left(N_{a_{i}}, L_{a_{i}}\right)_{i}\right)=\left(M_{a}, i d\right)$ for some $m$ and some nonzero $R$-module $M_{a}$ and $C H^{l}\left(\left(N_{a_{i}}, L_{a_{i}}\right)_{i}\right)=0$ for all $l \neq m$ then the preimage of each periodic orbit in $\Sigma_{A}$ contains a periodic orbit for $f$ with the same period.

The proofs for Theorem 4.1 and Corollary 4.2 are very similar to that of Theorem 1 and Corollary 3 in [Sr], therefore they are omitted. Details can be found in [Gi3].

Compared to Theorem 1.1 in [CaKwMi], this corollary produces a semi-conjugacy of $(S, f)$ (rather than $\left(S, f^{d}\right)$ for some $d$ ) and a shift-space, providing a more detailed description of the dynamics. Compared to Corollary 3 in [Sr], it has the advantage of using data independent of the choice of compact pairs and invariant under small perturbations of the map. 
We restate the above corollary in an over-restrictive but easier to apply form:

Corollary 4.3. Let $\left\{B_{i}\right\}_{i \in\{1, \ldots, \kappa\}}$ a countable collection of pairwise disjoint compact sets satisfying

$$
f^{-1}\left(B_{i}\right) \cap B_{j} \cap f\left(B_{l}\right) \subseteq i n t B_{j} \text { for all } i, j, l \in\{1, \ldots, \kappa\}
$$

Define the matrix $A=\left(\alpha_{i j}\right)_{i, j \in\{1, \ldots \kappa\}}$ by

$$
\alpha_{i j}= \begin{cases}1 & \text { if } f\left(B_{i}\right) \cap B_{j} \neq \emptyset \\ 0 & \text { otherwise }\end{cases}
$$

For every $i, j, l \in\{1, \ldots \kappa\}$ satisfying $\alpha_{i j} \alpha_{j l}=1$ define $N_{i}:=B_{i}, L_{i}:=B_{i} \backslash f^{-1}\left(\right.$ int $\left.B_{j}\right)$, $N_{j}:=B_{j}, L_{j}:=B_{j} \backslash f^{-1}\left(\right.$ int $\left.B_{l}\right)$ and $F_{j i}^{*}: H^{*}\left(N_{i}, L_{i}\right) \longrightarrow H^{*}\left(N_{j}, L_{j}\right)$ the transfer map as constructed in Section 2.

Assume that

$$
H^{k}\left(N_{i}, L_{i}\right)=H^{k}\left(N_{j}, L_{j}\right)=\left\{\begin{array}{lc}
R & \text { for } k=m \\
0 & \text { otherwise }
\end{array}\right.
$$

and

for some $m \geq 0$.

$$
F_{j i}^{k}= \begin{cases}i d & \text { for } k=m \\ 0 & \text { otherwise }\end{cases}
$$

Then $\left\{B_{a_{i}}\right\}_{i}$ is an isolating neighborhood sequence for every $a \in \Sigma_{A}, \operatorname{Con}^{k}\left(\left\{B_{a_{i}}\right\}_{i}\right)=$ $(R, i d)$ for $k=m$ and $\operatorname{Con}^{k}\left(\left\{B_{a_{i}}\right\}_{i}\right)=0$ for $k \neq m$ and there exists an invariant set $S \subseteq B_{1} \cup \ldots \cup B_{\kappa}$ and a semi-conjugacy $\phi: S \rightarrow \Sigma_{A}$ such that $\phi \circ f=\sigma_{A} \circ f$ and the preimage of each periodic orbit for $\sigma$ contains a periodic orbit for $f$.

EXAMPLE 4.4. We will apply Corollary 4.3 to study the dynamics on a topological horseshoe with singularities. Again, we consider the square $Q$ of vertices $A, B, C, D$ and a continuous map $f$ defined everywhere except the top edge $C D$ which acts as follows. First, it stretches the square in the vertical direction keeping the base $A B$ fixed and shifting the top $C D$ to infinity. Second, it shrinks non-uniformly each horizontal leaf: the more the square gets stretched in the vertical direction, the more it gets shrunk in the horizontal direction. Third, it folds the resulting strip infinitely many times, as it would follow the shape of the graph of $\sin (1 / x)$, approaching the horizontal left-hand side edge, without ever touching it. Thus, $Q \cap f(Q)$ consists of countably many rectangles of length converging to zero. The inverse map $f^{-1}$ is defined everywhere except the left-hand side edge $A D$ of the square. Thus, $f^{-1}(Q) \cap Q$ consists of countably many rectangles of width converging to zero. See Figure 4.1.

Let us denote the collection of these latest rectangles by $\mathcal{V}=\left\{V_{0}, V_{1}, V_{2}, \ldots\right\}$. The map $f$ extends to a homeomorphism of the two dimensional sphere $S^{2}$ the union of the edges $A D$ and $C D$. Let $\mathcal{S}$ denote the set of the singularities of $f$. The invariant set $S$ of $f$ is a subset of $S^{2} \backslash \bigcup_{i \in \mathbf{Z}} f^{-i}(\mathcal{S})$, it has infinitely many components and it may or may be not a Cantor set. There exist points in $S$ arbitrarily close to the set of singularities $\mathcal{S}$. This type of behavior is very similar to that of non-uniformly hyperbolic systems with singularities $[\mathrm{KrTr}]$. However, we do not require any hyperbolicity of the system. We do require only:

$$
f^{-1}\left(V_{i}\right) \cap V_{j} \cap f\left(V_{k}\right) \subseteq i n t V_{j}
$$




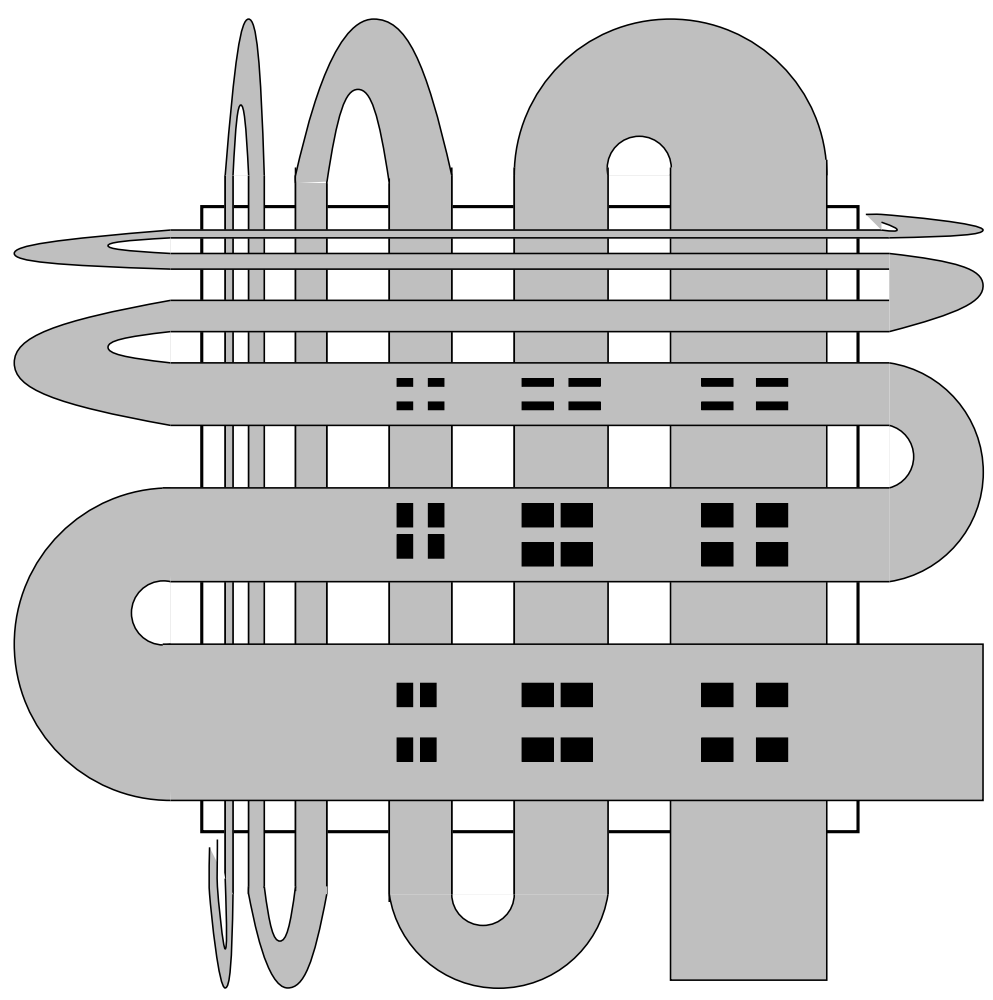

Fig. 4.1. The horseshoe with singularities

for all $i, j, k \in\{0,1,2, \ldots\}$

$$
\begin{gathered}
H_{\mathbf{Z}_{2}}^{k}\left(V_{i}, V_{i} \backslash f^{-1}\left(i n t V_{j}\right)\right)= \begin{cases}\mathbf{Z}_{2} & \text { for } k=2 \\
0 & \text { otherwise }\end{cases} \\
F_{j i}^{k}= \begin{cases}i d & \text { for } k=2 \\
0 & \text { otherwise }\end{cases}
\end{gathered}
$$

for all $i, j, \in\{0,1,2, \ldots\}$. We have chosen $\mathbf{Z}_{2}$ coefficients for simplicity.

Under the above conditions, by Corollary 4.3 we obtain the existence of a semiconjugacy $\phi: s \rightarrow \Sigma$ (where $\Sigma$ represents the full-shift space on symbols $\{0,1,2, \ldots\}$ ) with the properties that $\phi \circ f=\sigma \circ f$ and the preimage of each periodic orbit for $\sigma$ contains a periodic orbit for $f$.

Acknowledgments. This material is part of the author's thesis during his doctoral program at State University of New York at Buffalo. The author would like to express his gratitude to J. Reineck, K. Mischaikow, M. Mrozek and R. Srzednicki for their advice, comments and remarks.

\section{References}

[Bu] L. A. Bunimovich, A Theorem on Ergodicity of Two-Dimensional Hyperbolic Billiards, Comm. Math. Phys. 130 (1990), 599-621. 
[BuSi] L. A. Bunimovich and Ya. G. Sinai, Markov Partitions for Dispersed Billiards, Comm. Math. Phys. 78 (1980), 247-280.

[CaKwMi] M. Carbinatto, J. Kwapisz and K. Mischaikow, Horseshoes and the Conley Index Spectrum, preprint, CDSNS96-247.

[DeMr] J. Degiovanni and M. Mrozek, The Conley Index for Maps in the Absence of Compactness, Proc. Royal Soc. Edinburgh 123A (1993), 79-95.

[Gi1] M. GideA, The Discrete Conley Index for Non-Invariant Sets, Proceedings of the Topological Methods in Differential Equations and Dynamical Systems Conference, Kraków, Poland, July (1996), to appear.

[Gi2] M. GideA, Leray Functor and Orbital Conley Index for Non-Invariant Sets, Discrete and Continuous Dynamical Systems, to appear.

[Gi3] M. GideA, The Discrete Conley Index for Non-Invariant Sets and Detection of Chaos, thesis (1997).

[Gr] A. Granas, The Leray-Schauder Index and the Fixed Point Index for Arbitrary ANR's, Bull. Soc. Math. France 100 (1972), 209-228.

[KrTr] T. Kruger and S. Troubetzkoy, Markov Partitions and Shadowing for NonUniformly Hyperbolic Systems with Singularities, Erg. Th. Dyn. Sys. (1992), 487-508.

[Mi] K. Mischaikow, Conley Index Theory: Some recent development, preprint, CDSNS 94-181.

[MiMr1] K. Mischaikow and M. Mrozek, Chaos in Lorentz equation: A computer assisted proof, Bull. Amer. Math. Soc. 32 (1995), 66-72.

[MiMr2] K. Mischaikow and M. MrozeK, Isolating Neighborhoods and Chaos, Japan J. Indus. Appl. Math. 12 (1995), 205-236.

[Mr1] M. Mrozek, Leray functor and the cohomological Conley index for discrete dynamical systems, Trans. Amer. Math. Soc. 318 (1990), 149-178.

[Mr2] M. MrozeK, Index pairs and the fixed point index for semidynamical systems with discrete time, Fundamenta Mathematicae 133 (1989), 179-194.

[Sr] R. SRzednicki, A generalization of Lefschetz fixed point theorem and detection of chaos, preprint.

[Sp] E. H. Spanier, Algebraic Topology, McGraw-Hill, 1966.

[Sz1] A. SzymCZaK, The Conley index for decompositions of isolated invariant sets, Fundamenta Mathematicae 148 (1995), 71-90.

[Sz2] A. Szymczak, The Conley index for discrete semidynamical systems, Topology Appl., to appear. 